\title{
On the stick-slip waves under unilateral contact and Coulomb friction
}

\author{
H. D. Bui • A. Oueslati
}

\begin{abstract}
In this paper, the construction of analytic solutions of stick-slip waves propagating along the interface between an elastic half-space and a moving rigid one is investigated. The contact between the solids is governed by unilateral constraints and Coulomb friction law and the deformable body is loaded by remote uniform stresses $\tau_{y y}^{*}<0, \tau_{x y}^{*}>0$. The method of solution is based on the analytic continuation of Radok's complex potentials within the framework of steady elastodynamical problems. The governing equations combined with the boundary conditions are reduced to a Riemann-Hilbert problem with discontinuous coefficient. This approach for the stick-slip study is novel and differs from those in literature, namely the series method and the Weertman's dislocation formulation. We present the closed-form solution of the Riemann-Hilbert problem and show that the principal unknowns are of number two: the wave celerity and the ratio of the slip length by the stick one. The considered loading introduces an additional velocity $V^{*}$ related to the longitudinal elongation $\varepsilon_{x x}^{*}$ due to the normal stress $\tau_{y y}^{*}$. We show that if $V^{*}$ vanishes then there is no solution. When
\end{abstract}

\section{H. D. Bui}

Solid Mechanics Laboratory/CNRS UMR 7649,

Department of Mechanics, Ecole Polytechnique,

Palaiseau, France

e-mail: hdb@1ms.polytechnique.fr

H. D. Bui

Lamsid/CNRS UMR 2832, Electricité de France,

Clamart, France

\section{A. Oueslati}

Laboratoire de Mécanique de Lille/CNRS UMR 8107,

Université de Lille 1, UFR de Mathématiques Pures et

Appliquées, 59655 Villeneuve d'Ascq, France

e-mail: abdelbacet.oueslati@univ-lille1.fr
$V^{*} \neq 0$, it is possible to construct weakly singular solutions satisfying all stick-slip conditions except over a narrow zone at transition points: the shear stress is singular over a small zone and the normal contact stress exhibits a positive singularity over a very small zone in the slip region which implies a separation near the singular transition.

Keywords Stick-slip waves - Unilateral contact . Coulomb friction - Riemann-Hilbert problem . Crack-like behaviour

\section{Introduction}

Contact and friction are among the oldest branches of mechanics and continue to be a subject of intensive researches because of their crucial theoretical relevance and importance in engineering applications. Historically, it seems that the frictional contact theory started with the pioneering works of Amontons [7] and Coulomb [19] who formulated the well-known Coulomb friction law. This law is strongly nonlinear and nonsmooth because of its multivalued character and the presence of inequalities. Although Coulomb friction law is widely used in mechanics and engineering design many questions remains open such as the existence and uniqueness results of the solution [10, 14, 24, 29], theoretical convergence results and accuracy of numerical algorithms, shakedown of friction [6].

During the two last decades, an important issue emerges in the area of elastodynamic problems involving frictional contact, namely the friction-induced vibration resulting from the flutter instability in the spirit of Poincare-Hopf bifurcation. The induced vibrations have the form of stick-slip or stick-slip-separation self-sustained oscillations propagating along the contact interface and generally accompanied with 
noise emission. Many examples are common in daily life such as creaking door, noise of chalk against a table, brake squeal, guitar sound, silo music (sound emission during the flow of granular materials through silos) are generally associated to stick-slip propagation. Study of such periodic regimes is relevant for break squeal [31,38], simulations of earthquakes and seismology analysis [8, 11], study of ultrasonic motors [52], granular discharge from silos [35, 43], interpretation of Shallamach waves in sliding of rubber against a rigid substrate [12, 49].

In literature, first studies of stick-slip focused on discrete systems, typically the Van-der Pol or the Klarbring oscillator composed of a springs-mass assemblage in frictional contact with a rigid substrate moving with a constant velocity. For such mechanical systems, the formation of stick-slip motion is attributed to a static coefficient of friction higher than a kinematic one or to the decay of the kinematic coefficient with the sliding velocity. The transition toward friction induced vibration is numerically obtained in many works [40, 41]. The construction of analytical stick-slip solutions for discrete mechanical systems does not present any particular difficulty [22, 34].

Recent investigations of the steady sliding contact between dissimilar elastic half-spaces or between a semiinfinite elastic solid and a rigid one showed that the steady state is dynamically unstable for a constant friction coefficient. For instance, Renardy [47] explained the steady sliding contact between a rigid substrate and an incompressible half-plane within the framework of neo-Hooken constitutive behavior. He found that flutter instability occurs in the limit of elasticity for a coefficient of friction greater then 1. This same conclusion was established independently by Martins et al. [26, 27] and Simoes and Martins [48] after investigation of the dynamic response of an elastic and viscoelastic semi-infinite solids in contact with a rigid body. Further, they showed that the presence of viscous dissipation has the effect of increasing the minimum value of coefficient of friction required for existence of self-excited vibration. In the same spirit, surface instabilities in a Mooney-Rivlin half-space compressed against a rigid flat surface are studied by Désoyer and Martins [21] and it is found that the problem is again ill-posed for sufficiently large coefficients of friction. In a series of interesting papers, Adams [2-4] showed that the steady sliding between two dissimilar half-planes is ill-posed for a wide range of coefficient of friction, material combinations and relative sliding velocity. Adams [2] suggested that the dynamic instability of the steady state is related to the destabilization of the so-called interfacial slip waves. Such waves exist in frictionless contact and are called also the "generalized Rayleigh waves" because they propagate with the Rayleigh wave celerity. Recall that slip waves are initially observed by Weertman [51], Achenbach and
Epstein [1] and Murty et al. [36]. Later Rinjith and Rice [46] demonstrated the connection between the ill-posedness of the Coulomb friction problem and slip waves. Precisely, it was shown that, for material combinations where the generalized Rayleigh wave exists, the steady sliding with Coulomb friction is dynamically unstable for an arbitrarily small values of the friction coefficient. The authors showed that a possible regularization of the problem could be achieved by using a memory-dependent rateand-state friction law proposed by Prakash [44]. Nguyen et al. [32, 33, 39, 43] studied the sliding steady state for an elastic tube in frictional contact with a rigid and rotating shaft modeling a brake-like system. It is found that in frictionless contact, two harmonic waves propagating in opposite senses are obtained as in classical elasticity. When the Coulomb friction is considered, the relative sliding displacement represents two waves: an exploding wave and a damping one propagating in the opposite direction.

From the point of view of numerical studies, the illposedness of dynamic contact problems involving friction is encountered, for example, in works of Andrews et Benzion [8], Harris and Day [25] and Cochard and Rice [15] who investigated the propagation of ruptures under Coulomb friction along the interface of two dimensional and dissimilar solids. The ill-posedness manifests by the non convergence of numerical results with the refinement of grid-mesh. As suspected by Cochard and Rice [15] , the use of Parakash-Clifton's friction law for the same numerical investigations regularizes the problem.

Although the mechanism of flutter instability of friction is now well understood, only few works are devoted to the construction of analytic solution of self-excited vibrations for continuum media. First attempts to built closed-form solution of stick-slip or stick-slip-separation waves traveling along the interface contact between two identical elastic half-spaces, were derived by Comninou [16, 17]. Freund [23] pointed out a similarity feature between the Comninou's waves and the propagation of interface cracks and showed that the encountered singularities imply energy sources and sinks. Hence, Comninou's solutions were controversial and not physical. For these same reasons, Adams [3] didn't succeed to construct an interfacial stickslip waves between two dissimilar half-spaces, however, in Adams [4] a family of slip pulse propagating with the celerity of generalized Rayleigh waves is successfully built. Following the construction method of solution used by Comninou [17], Adams [3, 4] and Caroli [18] studied analytically the sliding between a viscoelastic half-space and nondeformable one. It is shown that the slow slippage between the bodies is impossible via a periodic set of alternating slip pulses and stick zones. Adda-Bedia and Ben Amar [5] studied the existence of a steady-state slip pulse on finite size between two dissimilar materials. In the 
presence of Coulomb friction, such solutions are found to exist with an arbitrary slip velocity and an arbitrary slip length. Nevertheless, these solutions are not physical and show a crack-like behavior because of the singularity of the normal contact stress. For bounded solids, to our knowledge, the only semi-analytic stick-slip and stick-slip-separation waves are derived in $[32,33,43]$ in view of the study of two-dimensional brake-like system. Based upon a Galerkin expansion, elastodynamic equations involving contact and Coulomb friction constraints are bring to the boundary giving a set of reduced equations. Resolution of the obtained reduced equations under contact and friction constraints allows the possibility of construction of traveling interface waves. According to the values of the contact pressure, the rotation velocity and the friction coefficient, several periodic dynamical responses can be found under the form of stick-slip and stick-slip-separation waves. These semi-analytic solutions are compared to numerical experimentations performed by finite element method [39, 42, 43]. Others numerical experimentations are provided in the literature [28, 30, 50].

The present work is devoted to the construction of an analytic solution of stick-slip waves crossing the interface of an elastic half-space and a rigid one. This model revisits some models on stick-slip problems [3, 4, 18, 26]. This paper is organized as follows. In Sect. 2, we set the governing equations and the boundary conditions for the problem of the steady sliding between the tow half-spaces. In Sect. 3, we proceed to the construction of the solution by using the analytical continuation method of the complex Radok's potentials [45]. The problem of the stick-slip is then reduced to a Riemann-Hilbert problem with discontinuous coefficient and the closed-form expressions of the solution are derived. These formulae are used in Sect. 4 to obtain the final equations allowing the determination of the stick and slip regions and the wave celerity. Section 5 is concerned with the results and the discussion of the characteristics of the obtained stick-slip wave.

\section{Problem statement}

Consider an elastic solid, with shear modulus $G$ and waves velocities $c_{1}, c_{2}$ occupying the lower half space $\Omega^{-}$and sliding against the upper rigid half-space $\Omega^{+}$which moves to the right with velocity $V$, as shown in Fig. 1, in plane strain conditions. Unilateral contact and Coulomb friction with constant friction coefficient $\mu$ are assumed. It is emphasized that the interfacial or local coefficient of friction $\mu$ is the ratio of shear to normal contact pressure at the interface which would cause local slipping to occur [4]. The elastic body is subjected to remote constant stresses $\tau_{y y}^{*}<0, \tau_{x y}^{*}>0$ such that $\tau_{x y}^{*}=-\mu^{*} \tau_{y y}^{*}$, with $\mu^{*}<\mu$.

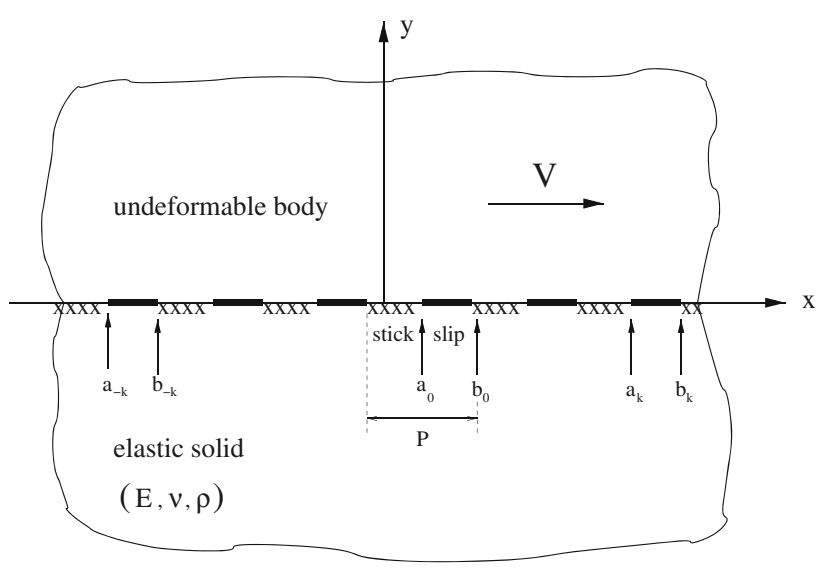

Fig. 1 A rigid body sliding on an elastic half-space

Suppose that the interface is entirely a stick zone, then the velocity of points of the interface $y=0^{-}$, is $\dot{U}_{x}=V$. Since the shear stress grows indefinitely, the interface will no longer adhere to the upper non-deformable solid.

On the other hand, suppose that the interface $y=0^{-}$is entirely a sliding one, with the constant static stress $\left(\tau_{x y}^{*}>0, \tau_{y y}^{*}<0\right)$. Thus the elastic body is at rest under static stress, $\dot{U}_{x}=0$. The static stress state violates the Coulomb friction law. Therefore both conditions on the velocity and Coulomb's friction law are incompatible. Thus, we investigate here the possibility of relative motion of the two bodies, due to the existence of periodic stick and slip regions which propagate along the interface with some wave speed $c$. In this case the quantity $\mu^{*}$ may be interpreted as the apparent coefficient of friction, since sliding occurs with that ratio of applied shear to normal traction [4, 5, 18].

There are many physical phenomena in Nature in which incompatibility exists and results in what is called "frustration". The best example is the following one. Let us consider a unit volume of liquid water inside a nondeformable container at room temperature. At a temperature lower than $0^{\circ} \mathrm{C}$, water freezes into solid ice under atmospheric pressure. Since the specific volume of ice is greater than that of liquid water about $10 \%$, the water being constrained by the container cannot freely transform into solid ice. Suppose now that the container is deformable with a bulk deformation $\theta^{*}=5 \%$ at zero ${ }^{\circ} \mathrm{C}$. Water will be transformed into a mixture of $50 \%$ liquid and $50 \%$ solid, which is a kind of "sorbet". To accommodate incompatibility or frustration between different conditions, Nature invents "homogenization", Aubry [9].

We assume that the periodic stick-slip wave consists in a stick region plus a slip one indefinitely repeated as shown in Fig. 1. Let us denote by $S L$ the set of the slip segments $S L=\cdots\left[a_{-k}, b_{-k}\right] \bigcup \cdots\left[a_{0}, b_{0}\right] \bigcup \cdots\left[a_{k}, b_{k}\right] \bigcup \cdots, \quad k \in \mathbb{N}$ 
The remainder part of contact interface is the stick zone and will be refereed by $S T$. The infinite axis $y=0$ is oriented in the direction of increasing $x$ and the following notation will be frequently used: $S L^{+}=\lim _{y \rightarrow 0^{+}} S L$ and $S L^{-}=$ $\lim _{y \rightarrow 0^{-}} S L$. In the same way, one defines $S T^{+}$and $S T^{-}$.

If we fix the origin of coordinate frame at $b_{-1}=0$, we can simply denote any particular slip zone $\left[a_{i}, b_{i}\right]$ by its generic segment $\left[a \equiv a_{0}, b \equiv b_{0}\right]$ and any stick zone $\left[b_{k-1}\right.$, $a_{k}$ ] by its generic segment $\left[b_{-1}=0, a \equiv a_{0}\right]$.

Together with a fixed frame coordinates $(X, Y)$, we shall use also moving coordinates $x=X-c t, Y=y$ where $c$ is the wave velocity. Material derivative in steady state case is denoted by a dot, $\frac{d g}{d t} \equiv \dot{g}=-c g_{, x}$.

In the present work, it is reasonable to assume that the periodic stick-slip regime occurs under the condition discussed above $\dot{U}_{x}=0$ which is satisfied, not locally in the whole interface, but only in the homogenized sense. That is the "mean" value of the velocity over a stick-slip period $[0, b]$ vanishes

$$
\left\langle\dot{U}_{x}\right\rangle=0
$$

This vanishing mean value ensures that the stress field of the lower elastic body does not blow up in time.

In the steady state case, Eq. (1) is equivalent to $\left\langle-c U_{x, x}\right\rangle=0$ or to the periodicity condition

$U(b)=U(0)$

Condition $\dot{U}_{x}=V$ is only satisfied in the stick zones $S T$, while the sliding condition $\Sigma_{x y}[U]=-\mu \Sigma_{y y}[U]$ is only satisfied in the slip zones $S L$. The incompatibility between boundary conditions is relaxed through homogenization so that the effective or homogenized "friction" coefficient $\mu^{*}$ can be smaller that the true one.

Let the material velocity in the elastic body at interface be decomposed into the sum

$\dot{U}=V^{*}+\dot{u}=V^{*}-c u_{x, x}$

where $V^{*}$ is some velocity defined hereafter. We shall show that the presence of longitudinal elongation $\varepsilon_{x x}^{*}$ enables to solve the periodic stick-slip problem. If this elongation is ignored by setting $\varepsilon_{x x}^{*}=0$ in the equation, we will show that there is no solution. From elastic law, the elongation in the $x$-direction is

$\varepsilon_{x x}^{*}=u_{x, x}^{*}=-\frac{v(1+v)}{E} \tau_{y y}^{*}>0$

The velocity $V^{*}$ is defined hereafter by

$V^{*}=-c u_{x, x}^{*}=c \frac{v(1+v)}{E} \tau_{y y}^{*}$

Note that $V^{*}$ is positive because $c<0$ as it will be shown hereafter. Equation (5) establishes a relationship between $\tau_{y y}^{*}, c$ and $V^{*}$. Hence, the principal unknowns are the wave celerity $c$ and $b$ for prescribed stresses and $V^{*}$.

Let $\sigma_{x y}, \sigma_{y y}$ and $\sigma_{x x}$ be the additional stresses correspondent to the perturbed stick-slip motion. The solution satisfies the following conditions

- Active contact along the interface $y=0$ :

$u_{y}=0$

- Material velocity:

$\dot{U} \equiv-c u_{x, x}+V^{*}=V \quad$ in the stick region $S T$

- Sliding condition:

$$
\begin{aligned}
& \Sigma_{x y}[U] \equiv \sigma_{x y}+\tau_{x y}^{*}=-\mu\left(\sigma_{y y}+\tau_{y y}^{*}\right) \equiv-\mu \Sigma_{y y}[U] \\
& \quad \text { in the slip region } S L
\end{aligned}
$$

- The following inequality:

$\left|\Sigma_{x y}[U]\right|<\mu\left|\Sigma_{y y}[U]\right|$ in the stick zone $S T$

- $\sigma_{x y}$ and $\sigma_{y y}$ behaves like

$\sigma_{i j}=O\left(1 /\left(x^{2}+y^{2}\right)\right) \quad$ at infinity

We search $c, \nabla u, \sigma_{x y}, \sigma_{y y}$ periodic satisfying relations (6-10). An addition condition comes from the periodicity of fields. Note that even if the following fields $\dot{U}$, $U$ (displacement), $\nabla u, \sigma$ are periodic, the field $u_{x}$, is not. A counter-example can be seen in Fig. 2 for the periodic field (continuous line) $u_{x, x}=1$ in $[0,1], u_{x, x}=-1$ in [1, $b \neq 0$ ] and the non-periodic continuous field $u_{x}$ (doted line).

From Eq. (1) we get

$-c U_{x, x}=-c u_{x, x}+V^{*}$

Assuming the periodicity condition for the total displacement $U(x)$

$U_{x}(b)-U_{x}(0)=0$

we get $-c\left(U_{x}(b)-U_{x}(0)\right)=-c\left(u_{x}(b)-u_{x}(0)\right)+V^{*} b=$ 0 and thus

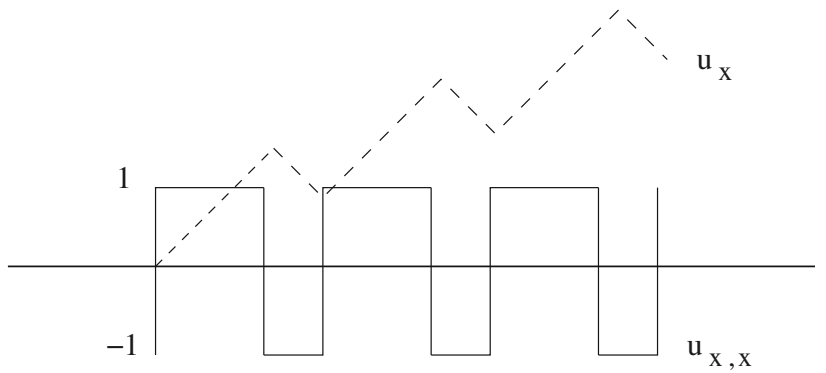

Fig. 2 The gradient field $u_{x, x}$ is periodic (continuous line) however the displacement $u_{x}$ is non-periodic (doted line) 
$u_{x}(b)-u_{x}(0)=\frac{b V^{*}}{c}$

Thus, $u_{x}(x, 0)$ is not periodic. The constant $-V^{*} / c$ has been introduced as the applied elongation $\varepsilon_{x x}^{*}$. Another interpretation of (13) is the sliding displacement over a period. Remark that Eq. (1) shows that the "mean perturbed velocity" $\langle\dot{u}\rangle$ over a period of length $b$ is opposite to $V^{*}$. Thus we recover (13)

$\langle\dot{u}\rangle=-\frac{c}{b}\left(u_{x}(b)-u_{x}(0)\right)=-V^{*}$

Another interpretation of Eq. (1) is to impose the overall "mean" strain $U_{x, x}$ equal to zero, or to require the "mean" strain $\left\langle u_{x, x}\right\rangle$ be opposite to the applied strain $u_{x, x}^{*}=-\frac{V^{*}}{c}$.

Let us now recall the steady elastodynamic equations within the framework of homogenous and isotropic elasticity under the plane strain hypothesis. In the moving frame $(O x y)$ attached to the propagating stick-slip wave $(x=X-c t, \quad y=Y)$, one introduces the Radok complex variables

$z_{1}=x+i \beta_{1} y \quad ; \quad z_{2}=x+i \beta_{2} y$

where $\beta_{1}=\sqrt{1-\frac{c^{2}}{c_{1}^{2}}}, \quad \beta_{2}=\sqrt{1-\frac{c^{2}}{c_{2}^{2}}}, \quad i=\sqrt{-1}$ is the imaginary unit number and $c_{1}, c_{2}$ stand for the celerity of longitudinal and shear waves defined respectively by

$c_{1}=\sqrt{\frac{\lambda+2 G}{\rho}}, \quad c_{2}=\sqrt{\frac{G}{\rho}}$

$G$ is the shear modulus, $\lambda$ denotes the Lamé's coefficient and $\rho$ is the mass density. The displacement and stress fields are given in terms of tow complex potentials $\phi_{1}$ and $\phi_{2}$ as follows [45]

$u_{x}=-\frac{1}{G} \Re e\left(\phi_{1}\left(z_{1}\right)+\frac{1+\beta_{2}^{2}}{2} \phi_{2}\left(z_{2}\right)\right)$

$u_{y}=\frac{1}{G} \Im m\left(\beta_{1} \phi_{1}\left(z_{1}\right)+\frac{1+\beta_{2}^{2}}{2 \beta_{2}} \phi_{2}\left(z_{2}\right)\right)$

$\sigma_{x x}=-2 \Re e\left(\frac{2 \beta_{1}^{2}-\beta_{2}^{2}+1}{2} \phi_{1}^{\prime}\left(z_{1}\right)+\frac{1+\beta_{2}^{2}}{2} \phi_{2}^{\prime}\left(z_{2}\right)\right)$

$\sigma_{y y}=\left(1+\beta_{2}^{2}\right) \Re e\left(\phi_{1}^{\prime}\left(z_{1}\right)+\phi_{2}^{\prime}\left(z_{2}\right)\right)$

$\left.\sigma_{x y}=2 \Im m \quad \beta_{1} \phi_{1}^{\prime}\left(z_{1}\right)+\frac{\left(1+\beta_{2}^{2}\right)^{2}}{4 \beta_{2}} \phi_{2}^{\prime}\left(z_{2}\right)\right)$

where $\Re e(Z)$ and $\Im m(Z)$ represent respectively the real and the imaginary part of the complex number $Z$. The present study focus on the subsonic waves i.e. $c<c_{2}$. Hence, $\beta_{1}>0$ and $\beta_{2}>0$.

\section{Construction method of the solution}

\subsection{Basic equations}

Following Bui and Oueslati [14] and Bui [13], the construction method of the solution is based on the displacement continuation. From the condition $u_{y}=0$ along the contact interface (real axis where $z_{1}=z_{2}=z=x+i 0$ ) and Eq. (16) one obtains

$\beta_{1} \phi_{1}(z)+\frac{\left(1+\beta_{2}^{2}\right)}{2 \beta_{2}} \phi_{2}(z)=0$

This equation suggests the following definition for the function $\phi_{2}$

$\phi_{2}\left(z_{2}\right):=-\frac{2 \beta_{1} \beta_{2}}{\left(1+\beta_{2}^{2}\right)} \phi_{1}\left(z_{2}\right)$

Substitution of (21) in (15-19) results in the following equations on the interface

$u_{x}=-\frac{\left(1-\beta_{1} \beta_{2}\right)}{G} \Re e\left(\phi_{1}(z)\right)$

$\sigma_{y y}=\left(1+\beta_{2}^{2}-2 \beta_{1} \beta_{2}\right) \Re e\left(\phi_{1}^{\prime}(z)\right)$

$\sigma_{x x}=\left(\left(-1+\beta_{2}^{2}\right)+2 \beta_{1}\left(\beta_{2}-\beta_{1}\right)\right) \Re e\left(\phi_{1}^{\prime}(z)\right)$

$\sigma_{x y}=\beta_{1}\left(1-\beta_{2}^{2}\right) \Im m\left(\phi_{1}^{\prime}(z)\right)$

Hence, all mechanical fields are determined through the knowledge of the function $\phi_{1}$ and its derivative $\phi_{1}{ }^{\prime}$.

In the sequel, unless stated otherwise, the notation $\Phi(z):=\phi_{1}^{\prime}(z)$ will be used.

We shall search for a complex solution under the form of a Cauchy integral with a distribution $f(t)$

$\Phi(z)=\frac{1}{2 i \pi} \int_{a}^{b} \frac{f(t)}{t-z} d t$

It is useful to underline that if $f(t)$ is real then the conjugate function of $\Phi$ is given by

$\bar{\Phi}(z)=-\frac{1}{2 i \pi} \int_{a}^{b} \frac{f(t)}{t-z} d t=-\Phi(z)$

Note that (27) may remains valid for some complex functions $f(t)$. It will be the case for the solution in the slip zone as it will be shown hereafter.

\subsection{Solution in the slip zone}

The sliding state expressed by Eq. (8) may be written as $\sigma_{x y}+\tau_{x y}^{*}=-\mu\left(\sigma_{y y}+\tau_{y y}^{*}\right) \Rightarrow \sigma_{x y}+\mu \sigma_{y y}=-\tau_{x y}^{*}-\mu \tau_{y y}^{*}:=T^{*}$ 
The stress $T^{*}$ can be written as $T^{*}=\mu^{*} \tau_{y y}^{*}-\mu \tau_{y y}^{*}=$ $\left(\mu^{*}-\mu\right) \tau_{y y}^{*}>0$. It is positive because $\mu^{*}<\mu$ and $\tau_{y y}^{*}<0$. By setting $\gamma_{1}=\beta_{1}\left(1-\beta_{2}^{2}\right)>0$ and $\gamma_{2}=1+\beta_{2}^{2}-2 \beta_{1} \beta_{2}$ and by virtue of $\bar{\Phi}\left(z^{+}\right)=-\Phi\left(z^{+}\right)$, Eq. (28) becomes

$-g \Phi\left(z^{-}\right)+\Phi\left(z^{+}\right)=\frac{2 i T^{*}}{\gamma_{1}-i \mu \gamma_{2}}:=f_{2}(t)$

where

$f_{2}(t)=\frac{2 i T^{*}}{\gamma_{1}-i \mu \gamma_{2}}$

is a constant and

$g=-\frac{\gamma_{1}+i \mu \gamma_{2}}{\gamma_{1}-i \mu \gamma_{2}}$

It is useful to notice that the coefficient $g$ can be expressed in a different manner

$g=-e^{2 i \pi \alpha}=e^{2 i \pi\left(\alpha+\frac{1}{2}\right)}, \quad|g|=1$

with

$\tan (\alpha \pi)=\mu \frac{\gamma_{2}}{\gamma_{1}}$

Equation (29) is called the Riemann-Hilbert problem and has a closed form solution [37]. The appropriate P-periodic solution of the homogenous Riemann-Hilbert problem has the following form (Appendix 1)

$X(z)=\prod_{k=-\infty}^{+\infty}(z-(a+k b))^{\frac{1}{2}-\alpha}(z-(k+1) b)^{-\frac{1}{2}+\alpha}$

involving product of holomorphic functions with a cut along the slip segments $S L$. Observe that $X(z)$ is regular at $a_{k}=a+k b$ and weakly singular at $b_{k}=(k+1) b .{ }^{1}$

It easily seen that

$$
\begin{aligned}
X(z) & =\lim _{N \rightarrow+\infty} \underbrace{\sum_{k=-N}^{+N}(z-(a+k b))^{\frac{1}{2}-\alpha}(z-(k+1) b)^{-\frac{1}{2}+\alpha}}_{X^{N}(z)} \\
& =\lim _{N \rightarrow+\infty} X^{N}(z)
\end{aligned}
$$

It can be proved for a fixed $z$, that the sum $e$ of the remaining terms is of order $e=O(1 / N)$. Thus $X^{N}(z)$ tends absolutely to a limit, denoted by $X(z)$ when $N \rightarrow \infty$.

Following Mushkhelishvili [37], the general solution of (29) has the form

\footnotetext{
${ }^{1}$ Regular functions at $a_{k}$ and $b_{k}$ are not considered for the construction of the solution because the infinite product $X(z)$ is divergent at infinity and thus the proposed approach does not work.
}

$\Phi^{s l i p}(z)=\frac{f_{2}}{2 i \pi} X(z)\left\{\int_{S L^{+}} \frac{1}{X\left(t^{+}\right)} \frac{d t}{t-z}+C_{2}\right\}$

where $C_{2}$ is an arbitrary constant. Besides, it is easy to prove that (36) satisfies $\bar{\Phi}(z)=-\Phi(z)$.

\subsection{Solution in the stick zone}

Equating the slip velocity of stick zone to $V-V^{*}$, $u_{x, x}=\frac{\partial u_{x}}{\partial x}=-\frac{\left(V-V^{*}\right)}{c}$, and knowing that $\frac{\partial(\cdot)}{\partial x}=\frac{\partial(\cdot)}{\partial z}+\frac{\partial(\cdot)}{\partial \bar{z}}$ we obtain

$$
\begin{aligned}
u_{x, x} & =-\frac{\left(1-\beta_{1} \beta_{2}\right)}{2 G}\{\Phi(z)+\Phi(\bar{z})\} \\
& =-\frac{\left(1-\beta_{1} \beta_{2}\right)}{2 G}\left\{\Phi\left(z^{-}\right)-\Phi\left(z^{+}\right)\right\}=-\frac{\left(V-V^{*}\right)}{c}
\end{aligned}
$$

The Plemelj formulas permit one to obtain the density function $f_{1}(t)$

$f_{1}(t)=\Phi\left(t^{+}\right)-\Phi\left(t^{-}\right)=-\frac{2 G\left(V-V^{*}\right)}{c\left(1-\beta_{1} \beta_{2}\right)}$

Note that $f_{1}(t)$ is a real constant. One possible solution is given by

$\Phi(z)=\frac{f_{1}}{2 i \pi} \int_{S T} \frac{d t}{t-z}$

With this form of solution, the displacement at infinity should be logarithmic, and not of order $O(1 / z)$ as required and thus does not respect the continuity condition on $y=0$. Thus the expression of $\Phi$ given by (39) must be modified. An adequate choice of a P-periodic, holomorphic and multivalued function is given by

$X(z)=\prod_{k=-\infty}^{+\infty}(z-(a+k b))^{\frac{1}{2}-\alpha}(z-(k+1) b)^{-\frac{1}{2}+\alpha}$

where $\alpha$ is a real number introduced in the previous section by the Eq. (33). Notice that this function is continuous through the slip segments, satisfies $X\left(z^{+}\right)=X\left(z^{-}\right)$for every $z \in S L$ and is constant at infinity (equal to 1 ). It then follows

$\left\{\frac{\Phi\left(z^{+}\right)}{X\left(z^{+}\right)}-\frac{\Phi\left(z^{-}\right)}{X\left(z^{-}\right)}\right\}=-\frac{2 G\left(V-V^{*}\right)}{c\left(1-\beta_{1} \beta_{2}\right)} \frac{1}{X\left(z^{+}\right)}$

Therefore, the expression of complex potential in the stick zone is given by [37]

$\Phi^{\text {stick }}(z)=\frac{f_{1}}{2 i \pi} X(z)\left\{\int_{S T^{+}} \frac{d t}{X\left(t^{+}\right)(t-z)}+C_{1}\right\}$

where $C_{1}$ is constant. 


\subsection{Stick-slip solution}

The stick-slip potential is obtained by sum the two complex solutions $\Phi^{\text {stick }}$ and $\Phi^{\text {slip }}$. We obtain the following Cauchy integral with discontinuous density

$$
\begin{aligned}
\Phi(z)= & \frac{1}{2 i \pi} X(z) \\
& \times\left\{f_{1} \int_{S T^{+}} \frac{d t}{X\left(t^{+}\right)(t-z)}+f_{2} \int_{S L^{+}} \frac{d t}{X\left(t^{+}\right)(t-z)}+C_{0}\right\}
\end{aligned}
$$

where $C_{0}$ is an arbitrary constant, $f_{1}$ and $f_{2}$ are constant given by (30) and (38) respectively. Moreover, since the stress field vanishes at infinity then $C_{0}=0$.

Explicit evaluation of $\Phi(z)$ requires the knowledge of the explicit expressions of the Cauchy integrals in (42). This is derived out in Appendix 2 and 3. We obtain

$\Phi(z)=f_{1} \frac{g}{g-1} i X(z) e^{-i \pi \alpha}\left(\frac{1}{Z(z)}-1\right)+\frac{f_{2}}{1-g}(1-X(z))$

where $Z(z)$ is defined by the same infinite product (34) but arranged differently, with cuts along stick zones ST (instead of cuts along slip zones for $X(z)$ )

$$
\begin{aligned}
Z(z)= & \cdots\left(z-b_{-(k-1)}\right)^{-\beta}\left(z-a_{-k}\right)^{\beta} \\
& \cdots \underbrace{\left(z-b_{(k-1)}\right)^{-\beta}\left(z-a_{k}\right)^{\beta}}_{Z_{k}(z)}\left(z-b_{k}\right)^{-\beta} \cdots
\end{aligned}
$$

where $\beta=\frac{1}{2}-\alpha$. It is important to emphasize that the function $Z$ satisfies the following relations

- On the stick zone $S T^{-}$

$$
Z(z)=-i e^{i \pi \alpha}\left|X\left(z^{-}\right)\right| \quad \text { and } \quad X(z)=\left|X\left(z^{-}\right)\right|
$$

- On the slip part $S L^{-}$

$$
Z(z)=\left|X\left(z^{-}\right)\right| \text {and } X(z)=i e^{-i \pi \alpha}\left|X\left(z^{-}\right)\right|
$$

Explicit calculation gives the following analytic expression of the stick-slip potential splitted in its real and imaginary parts

$$
\begin{aligned}
& \Phi(z \in S L)=\left\{-\frac{f_{1}}{2}(1-|X(z)|)+\frac{T^{*}}{\gamma_{1}} \cos (\pi \alpha)|X(z)|\right\} \\
& +i\left\{-\frac{f_{1}}{2} \mu \frac{\gamma_{2}}{\gamma_{1}}(|X(z)|-1)+\frac{T^{*}}{\gamma_{1}}(1-|X(z)| \sin (\pi \alpha))\right\}
\end{aligned}
$$

and

$$
\begin{aligned}
\Phi(z \in S T)= & -\frac{f_{1}}{2}-i\left\{-\frac{f_{1}}{2} \mu \frac{\gamma_{2}}{\gamma_{1}}+\frac{f_{1}}{2 \cos (\pi \alpha)}|X(z)|\right. \\
& \left.+\frac{T^{*}}{\gamma_{1}}(1-|X(z)|)\right\}
\end{aligned}
$$

\section{Some aspects of the stick-slip potential and final equations}

Complex functions given by (45) and (46) are weakly singular (i.e. square integrable) which avoids the problem of unbounded energy sources or sinks [23] at switching boundaries between stick and slip segments. Let us remark that the nature of the mathematical singularity was explored in Deng [20] and Bui and Oueslati [14] where it was shown that the singularity $\left(-\frac{1}{2}+\alpha\right)$ is associated to "push-in" test of rigid fibre in its elastic matrix material and the singularity $\left(\frac{1}{2}-\alpha\right)$ corresponds to "pull-out" kinematics.

Moreover, since $\Phi$ must behave as $O\left(1 / z^{2}\right)$ at infinity then terms of order $O(1 / z)$ must be canceled. This condition will be imposed for the truncated product $X^{N}(z)$ (a sufficient condition). Also, it will be shown that it is necessary to ensure the same kind of constraint over a single and an isolated generic stick-slip segment $\left[b_{-1}=0, a\right] \cup[a, b]$ (a necessary condition) as follows

$$
\begin{aligned}
& \int_{0}^{a} f_{1} \frac{d t}{\left(t^{+}-a\right)^{\frac{1}{2}-\alpha}\left(t^{+}-b\right)^{-\frac{1}{2}+\alpha}} \\
& \quad+\int_{a}^{b} f_{2} \frac{d t}{\left(t^{+}-a\right)^{\frac{1}{2}-\alpha}\left(t^{+}-b\right)^{-\frac{1}{2}+\alpha}}=0
\end{aligned}
$$

In order to identify the term of order $O(1 / z)$ of $\Phi^{s l i p}(z)$ at infinity, we shall rather consider the function $\Phi_{N}^{s l i p}(z)$ defined by

$$
\begin{aligned}
\Phi^{s l i p}(z) & =\lim _{N \rightarrow+\infty} \underbrace{\frac{f_{2}}{2 i \pi} X^{N}(z) \sum_{k=-N}^{+N} \int_{S L_{k}^{+}} \frac{1}{X^{N}\left(t^{+}\right)} \frac{d t}{t-z}}_{\Phi_{N}^{\text {slip }}(z)} \\
& =\lim _{N \rightarrow+\infty} \Phi_{N}^{s l i p}(z)
\end{aligned}
$$

where $S L_{k}^{+}=\left[a_{k}, b_{k}\right]+i 0$.

It is readily checked that

$\Phi_{N}^{s l i p}(z)=\frac{f_{2}}{1-g}\left(1-X^{N}(z)\right)$

According to Appendix 3, for $|z| \rightarrow \infty$ we have

$\Phi_{N}^{s l i p}(z)=-\frac{f_{2}}{1-g}(2 N+1) \frac{\beta}{z}(b-a)$

where $\beta=\frac{1}{2}-\alpha$.

In the same way, after some cumbersome calculus presented in Appendix 3, the complex potential $\phi_{\text {stick }}^{N}$ reads

$\Phi_{\text {stick }}^{N}(z)=f_{1} \frac{g}{g-1} i e^{-i \pi \alpha}\left(\frac{1}{Z^{N}(z)}-1\right)$

where $Z^{N}(z)$ is the truncated product of $Z(z)$. For $|z| \rightarrow \infty$, one obtains 
$\Phi_{N}^{\text {stick }}(z)=f_{1} \frac{g}{g-1} i e^{-i \pi \alpha}(2 N+1) \frac{\beta}{z}\left(b_{-1}-a\right)$

Considering equations (50) and (52) we obtain for large $|z|$

$$
\begin{aligned}
& \Phi_{N}^{\text {stick }}(z)+\Phi_{N}^{\text {slip }}(z) \\
& \quad=-\frac{\beta}{z} \frac{(2 N+1)}{g-1}\left\{f_{1} g i e^{-i \pi \alpha}\left(a-b_{-1}\right)+f_{2}(b-a)\right\}
\end{aligned}
$$

Therefore, canceling terms of order $O(1 / z)$ at infinity results in

$f_{1} g i e^{-i \pi \alpha}\left(a-b_{-1}\right)+f_{2}(b-a)=0$

or in an equivalent manner

$\frac{2 G\left(V-V^{*}\right)}{c\left(1-\beta_{1} \beta_{2}\right)}\left(\gamma_{1}+i \mu \gamma_{2}\right) e^{-i \pi \alpha}\left(a-b_{-1}\right)+2 T^{*}(b-a)=0$

It should be noted that this expression holds for any elementary wave and constitutes the explicit expression of (47).

Dividing (55) by $a$ and setting $b_{-1}=0$ (i.e. the stick segment starts at the origin $O$ of the coordinate frame) one obtains

$f_{1}\left(\gamma_{1}+i \mu \gamma_{2}\right) e^{-i \pi \alpha}-2 T^{*}(\delta-1)=0$

where $\delta=b / a$.

Equation (56) is apparently a complex one in the form $F_{1}(\delta, c)+i F_{2}(\delta, c)=0$, where $F_{1}$ and $F_{2}$ are real, so that it should be splitted into two real equations. However, exact calculus shows that $F_{2} \equiv f_{1}\left(-\gamma_{1} \sin (\alpha \pi)+\mu \gamma_{2} \cos (\alpha \pi)=\right.$ 0 because $\tan (\alpha \pi)=\mu \frac{\gamma_{2}}{\gamma_{1}}$. Equation (56) is thus reduced to $F_{1}(\delta, c)=0$ which provides the first relation between the wave velocity $c$ and $\delta$

$\frac{G\left(V-V^{*}\right)}{c\left(1-\beta_{1} \beta_{2}\right)}\left(\gamma_{1} \cos (\alpha \pi)+\mu \gamma_{2} \sin (\alpha \pi)\right)+T^{*}(\delta-1)=0$

The second relation between $c$ and $\delta$, which enables complete determination of these unknowns will be set further by examining the continuity of the tangential displacement (the normal component $u_{y}=0$ along the contact interface).

We start by determining the gradient displacement $u_{x, x}$ over the contact interface by setting

$u_{x, x}=-\frac{\left(1-\beta_{1} \beta_{2}\right)}{G} \Re e\{\Phi(z)\}$

Therefore, in the slip zone, Eq. (45) yields the following expression

$$
\begin{aligned}
u_{x, x}(z \in S L)= & -\frac{\left(V-V^{*}\right)}{c}+\left|X\left(z^{-}\right)\right| \\
& \times \underbrace{\left\{\frac{\left(V-V^{*}\right)}{c}-\frac{\left(1-\beta_{1} \beta_{2}\right)}{2 G} \cos (\pi \alpha) \frac{T^{*}}{\gamma_{1}}\right\}}_{\omega\left(c ; V-V^{*}, T^{*}\right)}
\end{aligned}
$$

The displacement $u_{x}$ over the generic slip segment $[a, b]$ is then obtained by the integration of (59)

$$
\begin{aligned}
u_{x}(x \in S L)= & D-\frac{\left(V-V^{*}\right)}{c} x \\
& +\omega\left(c ; V-V^{*}, T^{*}\right) \int_{a}^{x}\left|X\left(t^{-}\right)\right| d t
\end{aligned}
$$

where $D$ is an arbitrary constant.

The stick velocity is easily determined by the stick condition (37) or by virtue of (58) and (46) as in the slip case. One gets

$u_{x, x}(z \in S T)=-\frac{\left(V-V^{*}\right)}{c}$

Hence, the horizontal displacement in the generic stick segment $\left[b_{-1}=0, a\right]$ is given by

$u_{x}(x \in S T)=-\frac{\left(V-V^{*}\right)}{c} x+F$

where $F$ represents an arbitrary integration constant.

A simple inspection of the continuity condition at $a$ shows that $D=F$.

Also one must write the continuity condition obtained by periodicity of the total field namely, $U_{x}(b)=U_{x}(0)$, so that according to (2), $u_{x}(b)-u_{x}(0)=\frac{b V^{*}}{c}$. This permits one to have the second equation allowing complete determination of $c$ and $\delta$

$$
\begin{aligned}
-\frac{V \delta}{c}+ & \left\{\frac{\left(V-V^{*}\right)}{c}-\frac{1-\beta_{1} \beta_{2}}{2 G} \cos (\alpha \pi) \frac{T^{*}}{\gamma_{1}}\right\} \\
& \times \int_{a}^{b}\left|X\left(t^{-}\right)\right| d t=0
\end{aligned}
$$

The explicit calculation of this "closure" condition requires explicit determination of the integral $K=\int_{a}^{b}\left|X\left(t^{-}\right)\right| d t$. We obtain (Appendix 3)

$$
\int_{a}^{b}\left|X\left(t^{-}\right)\right| d t=\pi\left(\frac{1}{2}-\alpha\right)(b-a) \frac{1}{\cos (\pi \alpha)}
$$

Therefore, one deduces

$$
\begin{aligned}
& -\frac{V}{c} \delta+\left\{\frac{\left(V-V^{*}\right)}{c}-\left(1-\beta_{1} \beta_{2}\right) \cos (\pi \alpha) \frac{T^{*}}{2 G \gamma_{1}}\right\} \\
& \quad \times \pi\left(\frac{1}{2}-\alpha\right) \frac{(\delta-1)}{\cos (\pi \alpha)}=0
\end{aligned}
$$

In conclusion, the unknowns of this stick-slip problem $(c, \delta)$ for given loading conditions $\left(T^{*}, V, V^{*}\right)$ are completely determined by the set of the two Eqs. (57) and (65). 
Physical quantities involved in the problem may be scaled as follows

$$
\begin{gathered}
a=1, \quad \delta=\frac{b}{a} \rightarrow b>1, \quad \frac{T^{*}}{G} \rightarrow T, \quad \frac{V}{c_{2}} \rightarrow v, \\
\frac{V^{*}}{c_{2}} \rightarrow v^{*}, \quad \frac{c_{1}}{c_{2}} \rightarrow \bar{c}_{1}, \quad \frac{c}{c_{2}} \rightarrow \bar{c}
\end{gathered}
$$

We obtain the following final equations

$$
\begin{aligned}
& -\frac{v}{\bar{c}} b+\left\{\frac{\left(v-v^{*}\right)}{\bar{c}}-\left(1-\beta_{1} \beta_{2}\right) \cos (\pi \alpha) \frac{T}{2 \gamma_{1}}\right\} \\
& \quad \times \pi\left(\frac{1}{2}-\alpha\right) \frac{(b-1)}{\cos (\pi \alpha)}=0
\end{aligned}
$$

and

$$
\frac{\left(v-v^{*}\right)}{\bar{c}\left(1-\beta_{1} \beta_{2}\right)}\left(\gamma_{1} \cos (\alpha \pi)+\mu \gamma_{2} \sin (\alpha \pi)\right)+T(b-1)=0
$$

\section{Results and discussion}

Following results in literature [26, 27, 48], the stick-slip wave propagates from rear to front, i.e. from the right to the left in our problem. Therefore, we shall search for a wave celerity in the range $-c_{2}<c<0$ (or $-1<\bar{c}<0$ for the non dimensional equations).

The stick-slip problem $(\bar{c}, b)$, for given loading conditions $\left(T, v, v^{*}\right)$ is solved by the set of the two equations (66) and (67). This problem is highly non linear in the wave velocity $c$ and the uniqueness of the solution cannot be easily proved. Moreover its numerical solution by Newton's method for example, is unaccurate and fails because of singularities. Let us propose a simpler method to solve it. Instead of finding $(\bar{c}, b)$ for a given loading condition $\left(v, v^{*}, T\right)$ (or $\left(v, v^{*}, \tau_{x y}^{*}\right)$ since), we search the solution of the following inverse problem:

Find $(v, T)$ for any given pair $(\bar{c}, b)$ and givenv*.

Therefore, one obtains a linear algebric system

$$
\begin{aligned}
& {\left[\pi\left(\frac{1}{2}-\alpha\right) \frac{(b-1)}{\cos (\pi \alpha)}-b\right] \frac{v}{\bar{c}}} \\
& \quad-\left[\pi\left(\frac{1}{2}-\alpha\right)(b-1) \frac{\left(1-\beta_{1} \beta_{2}\right)}{2 \gamma_{1}}\right] T \\
& \quad=\pi\left(\frac{1}{2}-\alpha\right) \frac{(b-1)}{\cos (\pi \alpha)} \frac{v^{*}}{\bar{c}}
\end{aligned}
$$

and

$$
\begin{aligned}
& {\left[2 \frac{\left(\gamma_{1} \cos (\alpha \pi)+\mu \gamma_{2} \sin (\alpha \pi)\right)}{\left(1-\beta_{1} \beta_{2}\right)}\right] \frac{v}{\bar{c}}+[b-1] T} \\
& \quad=2 \frac{\left(\gamma_{1} \cos (\alpha \pi)+\mu \gamma_{2} \sin (\alpha \pi)\right)}{\left(1-\beta_{1} \beta_{2}\right)} \frac{v^{*}}{\bar{c}}
\end{aligned}
$$

The system (68-69) is mathematically well-posed in the sense that we have two equations for two unknowns $(v, T)$. Note that once $T$ is known the stresses $\tau_{y y}^{*}$ and $\tau_{x y}^{*}$ are easily obtained.
The solution is straightforward and depends on the determinant of the linear system

$D(b, \bar{c})=\frac{(b-1)}{\gamma_{1} \bar{c}} H(b, \bar{c})$

where

$$
\begin{aligned}
H(b, \bar{c})= & -\gamma_{1} b+\gamma_{1} \pi\left(\frac{1}{2}-\alpha\right) \frac{(b-1)}{\cos (\pi \alpha)} \\
& +\pi\left(\frac{1}{2}-\alpha\right)\left(\gamma_{1} \cos (\alpha \pi)+\mu \gamma_{2} \sin (\alpha \pi)\right)
\end{aligned}
$$

It is easy to establish that $H(b, \bar{c})>0 \forall b>1$ and $\forall \bar{c} \in]-1,0[$. Thus the determinant $D(b, \bar{c})$ is strictly negative $\forall b>1$ and $\forall \bar{c} \in]-1,0[$.

We shall distinguish two cases: $v^{*}=0$ and $v^{*} \neq 0$.

\subsection{Solution for $v^{*}=0$}

When $v^{*}=0$, equations (68-69) are reduced to a homogenous algebric system. The later have non trivial solutions in the domain $-1<\bar{c}<0$ and $b>1$ if and only if the determinant $D(b, \bar{c})$ vanishes. However, we have checked that $D(b, \bar{c})<0$ in the domain $-1<\bar{c}<0$ and $b>1$. Therefore, only the trivial solution $v=0$ (statics) and $T=0$ (the loading at the Coulomb limit) exists.

We conclude that no solution exists in the case $v^{*}=0$. This result agrees with the conclusion of Caroli [18] for friction of a viscoelastic medium against a rigid half-space where no solution is found.

\subsection{Solution for $v^{*} \neq 0$}

For the case $v^{*} \neq 0$ the solution is straightforward for $v$ and $T$

$v=\frac{v^{*}}{1+A(b, \bar{c})}$

where

$$
\begin{aligned}
& A(b, \bar{c}) \\
& \quad=\frac{4 b \gamma_{1} \cos (\alpha \pi)}{\pi(-1+2 \alpha)\left(\mu \gamma_{2} \sin (2 \pi \alpha)+\gamma_{1}(-1+2 b+\cos (2 \pi \alpha))\right)}
\end{aligned}
$$

and

$$
\begin{aligned}
T & =-\frac{4 b \gamma_{1}\left(\gamma_{1}+\gamma_{1} \cos (2 \alpha \pi)+\mu \gamma_{2} \sin (2 \alpha \pi)\right)}{(b-1)\left(-1+\beta_{1} \beta_{2}\right)} \\
& \times \frac{v^{*}}{\bar{c}\left(4 b \gamma_{1} \cos (\alpha \pi)+\pi(-1+2 \alpha)\left(\mu \gamma_{2} \sin (\pi \alpha)+\gamma_{1}(-1+2 b+\cos (\pi \alpha))\right)\right.}
\end{aligned}
$$

Note that the solution pair $(v, T)$ exists and is unique for any wave velocity such that $-1<\bar{c}<0$, any $b>1$ and for a given $v^{*}$. 
As can be noted in Eqs. (22) and (23), the gradient $u_{x, x}$ and the stress $\sigma_{y y}$ are of opposite signs. Since the slip velocity has a negative singularity at $x=b$ (Fig. 3), the normal stress shows a positive singularity as seen in Fig. 4 where $\Sigma_{y y} / G$ is plotted. Note also that, in the stick zone at the transition point with slip segments, the shear stress is singular and thus the stick condition (10) is violated over a very small zone, cf. Fig. 5. A similar result has been pointed out by Adda-Bedia and Ben Amar (2003) in analyzing the existence of a single steady-state slip pulse between two elastic media by the use of Weertman's dislocation formulation.

It is worth noting that the simplicity of the inverse problem allows an easy parametric study of sensibility of the results under various conditions. We may calculate $R=$ (separation region) $/ b$ (where $\Sigma_{y y}>0$ ) and $S$ the ratio of the zone length for which (10) is violated by $b$. They are found very small for a wide range of different combinations of the problem parameters. For example, for fixed

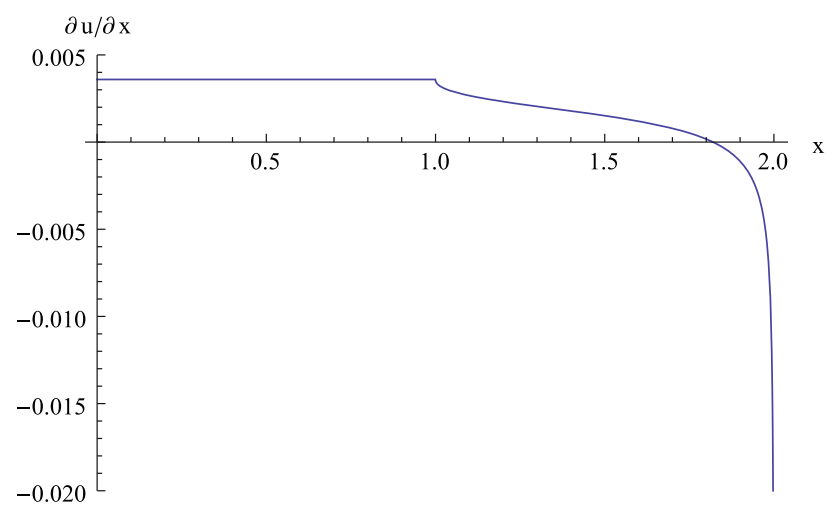

Fig. 3 The gradient $u_{x, x}$ over a stick-slip segment: a negative singularity near $b=2$ with $E=2.1 \times 10^{11} \mathrm{~Pa}, \rho=7,850 \mathrm{Kg} / \mathrm{m}^{3}$, $v=0.3, \mu=0.5, \mu^{*}=0.4, v^{*}=0.001$ and $\bar{c}=-0.7$

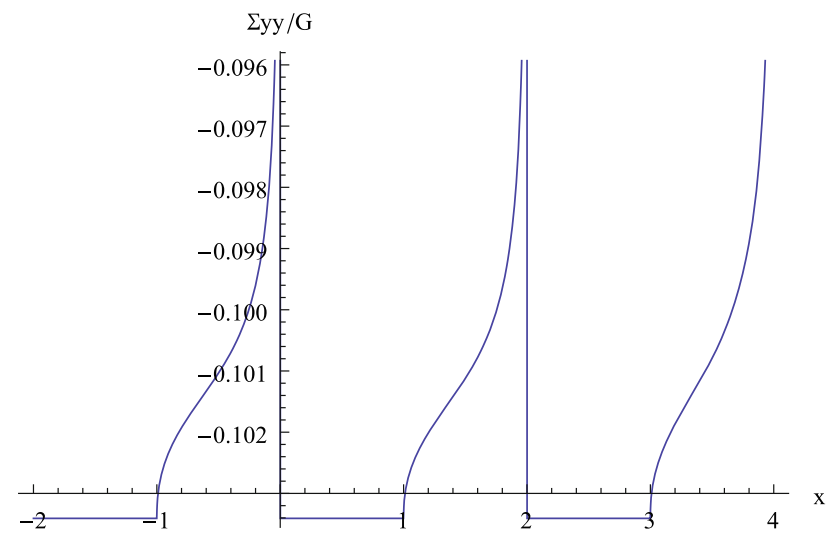

Fig. 4 The normalized stress $\Sigma_{y y} / G$ (for the same numerical data as for Fig. 3) over stick-slip segments exhibits a positive singularity at the transition points $S L \rightarrow S T$

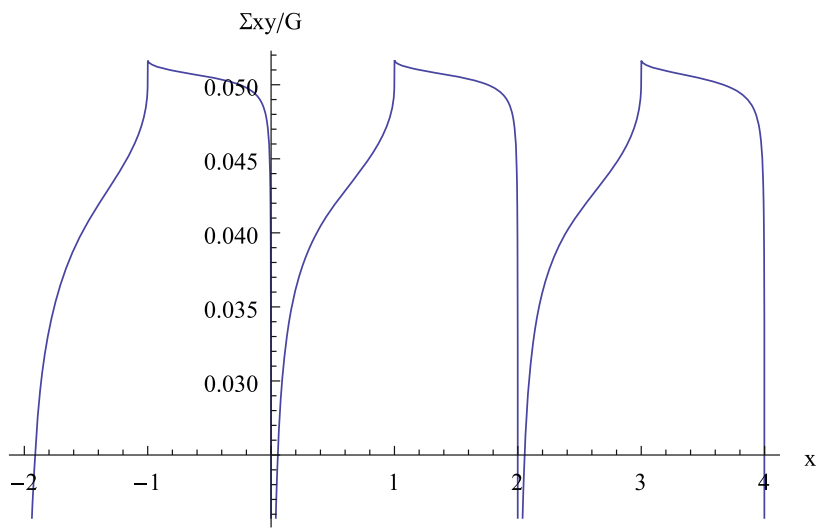

Fig. 5 The normalized stress $\Sigma_{x y} / G$ (for the same numerical data as for Fig. 3) over stick-slip segments shows a negative singularity at the transition points $S L \rightarrow S T$

$E=2.1 \times 10^{11} \quad \mathrm{~Pa}, \quad \rho=7,850 \mathrm{Kg} / \mathrm{m} 3, \quad v=0.3, \mu-$ $\mu^{*}=0.2, \quad v^{*}=0.001$ and $\bar{c}=-0.7$, it is found that $R(b=2)=6 \times 10^{-5}$ and $R(b=1.05)=3.1 \times 10^{-6}$ while $S(b=2)=3 \times 10^{-4}$ and $S(b=1.05)=6.3 \times 10^{-5}$. Besides for fixed $b=1.5, v^{*}=0.1, c=-0.7$ and $\mu=0.7$ these ratios vary with respect to $\mu^{*}$ as follows: $R\left(\mu^{*}=0.1\right)=10^{-3}$ and $R\left(\mu^{*}=0.65\right)=3.3 \times 10^{-6}$ while $S\left(\mu^{*}=0.1\right)=2 \times 10^{-2}$ and $S\left(\mu^{*}=0.65\right)=6.3 \times 10^{-5}$. Finally, the ratios $R$ and $S$ are practically not sensitive to variation of $v^{*}$ and $c$.

\section{Conclusion}

This work may be considered as a natural continuation of the results of many authors devoted mainly in the frictional slipping contact between two dissimilar media [26, 27, 46, 48] who showed that the steady sliding state is dynamically unstable which may lead to the formation of interface waves such as stick-slip or stick-slip-separation responses. We have shown that the stick-slip problem under conditions specified in the Introduction for the frictional sliding contact between an elastic medium a rigid one, can be solved analytically:

- The method of representation of plane strain steady state elastodynamics by Radok's potentials has been used.

- The stick-slip problem is reduced to a Riemann-Hilbert problem with discontinuous coefficient.

- The obtained equation have been solved in a closedform under the conditions that a uniform longitudinal strain $\varepsilon_{x x}^{*}=u_{x, x}^{*}$ is considered as the consequence of the normal stress $\tau_{y y}^{*}$. If the strain $\varepsilon_{x x}^{*}$ vanishes or is ignored $v^{*}=-c u_{x, x}=0$ there is no solution.

- The solution is obtained by solving an inverse procedure in which the loading conditions $(v, T)$ is 
determined as a function of the wave celerity $\bar{c}, b$ and $v^{*}$. There is no oscillatory behavior of the stress but the solution shows a crack-like behaviour: the shear stress exhibits a negative singularity while the normal stress has a positive singularity at the extremity of the slip region which also means a separation in a narrow zone. This incomplete solution is obtained for functions $X$ and $Z$ of the type "regular-weakly singular" at the ends $a_{k}$ and $b_{k}$ respectively.

Contrary to some authors who change the friction law and adopt generally the rate-and-state Prakash's friction law to overcome this difficulty, we will conserve the Coulomb friction law and investigate at least many possible models in the future: (1) Model of stick-slip-separation (2) Stick-slip-separation-reverse slip waves as already worked out semi-analytically and numerically by Oueslati [43] and Nguyen et al. [39] (3) Model of stick-slip with regular functions at $a_{k}$ and $b_{k}$, for which the method outlined in this paper does not work because the infinite product $\mathrm{X}(\mathrm{z})$ and $\mathrm{Z}(\mathrm{z})$ are divergent at infinity.

\section{Appendix 1: Periodic solution of the homogenous Riemann-Hilbert problem}

We search a P-periodic solution $(P=b-a)$ of the homogenous Riemann-Hilbert problem (29). First, a solution is derived for a generic slip segment $[a, b]$ and then is continued by periodicity to the whole slip region $S L$. Making use of Mushkhelishvili's results [37], the solution of the problem under consideration has the following form, with positive or negative $o d d$ integers $(n=-1,1,3, \ldots)$, $(m=-3,-1,1,3, \ldots)$ :

$Y(z)=(z-a)^{\frac{n}{2}-\alpha}(z-b)^{\frac{m}{2}+\alpha}$

with a cut along the slip segment $[a, b]$. Depending on the choice of odd integers $n, m$, these functions may be weakly singular (or square-integrable) or strongly singular (nonsquare-integrable). For physical reasons, strongly singular functions are not considered for the construction of the solution. Therefore, the appropriate fundamental solution of the homogenous problem is provided by:

$X_{0}(z)=(z-a)^{\frac{1}{2}-\alpha}(z-b)^{-\frac{1}{2}+\alpha}$

Unfortunately this function is not P-periodic as it is required. Note that the choice of the solution of the homogenous problem is very large because it depends on the polynomial function or in more general of an holomorphic function. As a matter of fact, we shall rather consider the following P-periodic function composed of an infinite product as a solution of the homogenous RiemannHilbert problem
$X(z)=\prod_{k=-\infty}^{+\infty}(z-(a+k b))^{\frac{1}{2}-\alpha}(z-(k+1) b)^{-\frac{1}{2}+\alpha}$

Appendix 2: Asymptotic expansion of $X^{N}(z)$ and $\frac{1}{X^{N}(z)}$ when $|z| \rightarrow \infty$

Consider the truncated product

$$
\begin{aligned}
X^{N}(z)= & (z-a)^{\beta}(z-b)^{-\beta} \\
& \times \prod_{k=-N, k \neq 0}^{k=N} \underbrace{(z-(a+k b))^{\beta}(z-(k+1) b)^{-\beta}}_{F_{k}(z)}
\end{aligned}
$$

where $\beta=\frac{1}{2}-\alpha$.

For very large $|z|$ we have:

$$
(z-a)^{\beta}(z-b)^{-\beta}=\left(1-\frac{a}{z}\right)^{\beta}\left(1-\frac{b}{z}\right)^{-\beta} \simeq 1+\beta \frac{(b-a)}{z}+\cdots
$$

and

$$
\begin{aligned}
F_{k}(z) & =\left(1-\frac{(a+k b)}{z}\right)^{\beta}\left(1-\frac{(k+1) b}{z}\right)^{-\beta} \\
& \simeq 1+\frac{\beta}{z}(b-a)+\cdots
\end{aligned}
$$

Hence, one obtains:

$$
\prod_{k=-N, k \neq 0}^{k=N} F_{k} \simeq\left(1+\frac{\beta}{z}(b-a)\right)^{2 N} \simeq 1+2 N \beta \frac{(b-a)}{z}+\cdots
$$

and consequently

$X^{N}(z) \simeq 1+(2 N+1) \beta \frac{(b-a)}{z}+\cdots$

Similar calculations are carried out for

$$
\begin{aligned}
\frac{1}{X^{N}(z)}= & (z-a)^{-\beta}(z-b)^{\beta} \\
& \times \prod_{k=-N, k \neq 0}^{k=N}(z-(a+k b))^{-\beta}(z-(k+1) b)^{\beta}
\end{aligned}
$$

and it is easily established that

$\frac{1}{X^{N}(z)} \simeq 1-(2 N+1) \beta \frac{(b-a)}{z}+\cdots=\alpha_{0}+\alpha_{-1} z^{-1}+\cdots$

where $\alpha_{0}=1$ and $\alpha_{-1}=-(2 N+1) \beta(b-a)$.

\section{Appendix 3: Evaluation of the integrals $I, J$ and $K$}

Integral $I=\frac{1}{2 i \pi} \int_{S L^{+}} \frac{d t}{X\left(t^{+}\right)(t-z)}$ 
The integral $I$ can be evaluated explicitly over the slip zone $S L^{+}$basing upon the asymptotic expansion of $1 / X(z)$ for large $|z|$. It is obvious that one has $X(z) \approx 1$ when $|z| \rightarrow \infty$ and hence [37]:

$I=\frac{1}{1-g}\left\{\frac{1}{X(z)}-\alpha_{0}\right\}$

where $\alpha_{0}=1$.

Therefore

$\Phi^{s l i p}(z)=f_{2} X(z) I=\frac{f_{2}}{1-g}(1-X(z))$

In a similar manner, we give the explicit expression of the integrale $I^{N}$ defined by:

$I^{N}(z)=\frac{1}{2 i \pi} \int_{S L^{+}} \frac{d t}{X^{N}\left(t^{+}\right)(t-z)}$

It turns out

$I^{N}(z)=\frac{1}{1-g}\left\{\frac{1}{X^{N}(z)}-\alpha_{0}\right\}$

where $g$ is given by expression (31) and $\alpha_{0}=1$.

Moreover, for $|z| \rightarrow \infty$, results established in Appendix 2 permit one to write:

$I^{N}=\frac{1}{1-g}\left\{\frac{1}{X^{N}(z)}-1\right\} \simeq-\frac{1}{1-g}(2 N+1) \beta \frac{b-a}{z}$

Integral $J=\frac{1}{2 i \pi} \int_{S T^{+}} \frac{d t}{X\left(t^{+}\right)(t-z)}$

The explicit knowledge of the solution potential $\Phi^{\text {stick }}(z)$ requires the explicit calculation of the integral $J$.

A particular care must be taken for this task since the function $X(z)$ is holomorphic in the hole space with a cut along slip segments $S L$ and therefore is continuous on stick zone $S T\left(X\left(z^{+}\right)=X\left(z^{-}\right) z \in S T\right)$. In order to evaluate explicitly the integral $J$ one has to introduce an holomorphic function in the complex plane with a cut along zones segments $S T$.

Let us introduce a new complex function $Z(z)$ as an infinite product of fundamental function $Z_{k}(z)$ obtained by a appropriate rearrangement of the function $X(z)=\cdots(z-$ $\left.b_{-(k-1)}\right)^{-\beta}\left(z-a_{-k}\right)^{\beta} \cdots\left(z-b_{(k-1)}\right)^{-\beta} \underbrace{\left(z-a_{k}\right)^{\beta}\left(z-b_{k}\right)^{-\beta}}_{X_{k}(z)} \cdots$ as follows

$$
\begin{aligned}
Z(z)= & \cdots\left(z-b_{-(k-1)}\right)^{-\beta}\left(z-a_{-k}\right)^{\beta} \\
& \cdots \underbrace{\left(z-b_{(k-1)}\right)^{-\beta}\left(z-a_{k}\right)^{\beta}}_{Z_{k}(z)}\left(z-b_{k)}\right)^{-\beta} \cdots
\end{aligned}
$$

where as usual $\beta=\frac{1}{2}-\alpha$.
$Z$ satisfies: $Z(z)=-i e^{i \pi \alpha}\left|X\left(z^{-}\right)\right|, z \in S T$ and $Z(z)=$ $\left|X\left(z^{-}\right)\right|$for $z \in S L^{-}$. The integral $J$ may be written as

$J=\frac{1}{2 i \pi} \int_{S T^{+}} \frac{d t}{X\left(t^{+}\right)(t-z)}=\frac{1}{2 i \pi} \int_{S T^{+}} \frac{i e^{-i \pi \alpha}}{Z\left(t^{+}\right)} \frac{d t}{t-z}$

On the one hand, by residue theorem ( $Z$ being equal to 1 at infinity) one has

$M(z)=\frac{1}{2 i \pi} \int_{S T^{+} \cup S T^{-}} \frac{1}{Z(t)} \frac{d t}{t-z}=\frac{1}{Z(z)}-1$

and on the other hand

$$
\begin{aligned}
M(z) & =\frac{1}{2 i \pi} \int_{S T^{+}} \frac{1}{Z\left(t^{+}\right)} \frac{d t}{t-z}+\frac{1}{2 i \pi} \int_{S T^{-}} \frac{1}{Z\left(t^{-}\right)} \frac{d t}{t-z} \\
& =\frac{1}{2 i \pi} \int_{S T^{+}} \frac{1}{Z\left(t^{+}\right)} \frac{d t}{t-z}+\frac{1}{2 i \pi} \sum_{k} \int_{b_{k}}^{a_{k}} \frac{1}{Z\left(t^{-}\right)} \frac{d t}{t-z} \\
& =\frac{1}{2 i \pi} \int_{S T^{+}} \frac{1}{Z\left(t^{+}\right)} \frac{d t}{t-z}-\frac{1}{2 i \pi} \sum_{k} \int_{a_{k}}^{b_{k}} \frac{1}{Z\left(t^{-}\right)} \frac{d t}{t-z} \\
& =\frac{1}{2 i \pi} \int_{S T^{+}} \frac{1}{Z\left(t^{+}\right)} \frac{d t}{t-z}-\frac{1}{2 i \pi} \sum_{k} \int_{a_{k}}^{b_{k}} \frac{1}{g Z\left(t^{+}\right)} \frac{d t}{t-z} \\
& =\left(1-\frac{1}{g}\right) \frac{1}{2 i \pi} \int_{S T^{+}} \frac{1}{Z\left(t^{+}\right)} \frac{d t}{t-z}
\end{aligned}
$$

Comparing (86)-(87) we get the integral $J$, Eq. (85), in explicit form

$$
\begin{aligned}
J & =\frac{1}{2 i \pi} \int_{S T^{+}} \frac{1}{X\left(t^{+}\right)} \frac{d t}{t-z}=\frac{i e^{-i \pi \alpha}}{2 i \pi} \int_{S T^{+}} \frac{1}{Z\left(t^{+}\right)} \frac{d t}{t-z} \\
& =\frac{i e^{-i \pi \alpha} g}{g-1}\left(\frac{1}{Z(z)}-1\right)
\end{aligned}
$$

The same method may be used for the evaluation of the integral $J^{N}$.

Integral $K=\int_{a}^{b}\left|X\left(t^{-}\right)\right| d t$

Let us start by $G^{N}=\frac{1}{2 i \pi} \int_{S L^{N}} \frac{d t}{X^{N}\left(t^{+}\right)}$.

Following Mushkhelishvili [37], it is readily checked that

$G^{N}=\frac{1}{2 i \pi} \int_{S L^{N}} \frac{d t}{X^{N}\left(t^{+}\right)}=-\frac{\alpha_{-1}}{1-g}$

where $\alpha_{-1}=-\beta \frac{(2 N+1)}{b-a}$ (see Appendix 2). Here we have taken the truncated product $X^{N}(z)$ for which $S L^{N}$ zone is composed by $(2 N+1)$ stick segments. Thanks to the 
periodicity of the solution, $G^{N}$ over the generic slip segment $[a, b]$ is given by:

$$
\begin{aligned}
\frac{1}{2 i \pi} \int_{a}^{b} \frac{d t}{X^{N}\left(t^{+}\right)} & =\frac{1}{2 i \pi} \frac{1}{(2 N+1)} \int_{S L^{N}} \frac{d t}{X^{N}\left(t^{+}\right)}=\frac{G^{N}}{2 N+1} \\
& =\beta \frac{(b-a)}{1-g}
\end{aligned}
$$

Consider now the function $S^{N}(z)=\frac{1}{X^{N}(z)}$. It satisfies the linear relationship $S^{N}\left(t^{-}\right)=g S^{N}\left(t^{+}\right)$. Similar procedure used for calculating $G^{N}$ can be applied for evaluating the integral $H^{N}$ over $(2 N+1)$ slip segments:

$H^{N}=\int_{S L^{N}} \frac{d t}{S^{N}\left(t^{+}\right)}=-\beta \frac{g}{g-1}(b-a)$

since one has

$$
\begin{aligned}
\frac{1}{S^{N}\left(t^{+}\right)} & =X^{N}\left(t^{+}\right)=g X^{N}\left(t^{-}\right)=g e^{i \beta \pi}\left|X^{N}\left(t^{-}\right)\right| \\
& =g e^{i \pi\left(\frac{1}{2}-\alpha\right)}\left|X^{N}\left(t^{-}\right)\right|=-i e^{i \pi \alpha}\left|X^{N}\left(t^{-}\right)\right|
\end{aligned}
$$

Therefore

$$
\begin{aligned}
\frac{1}{2 i \pi} \int_{a}^{b} X^{N}\left(t^{+}\right) d t & =\frac{1}{2 i \pi} \int_{a}^{b}-i e^{i \pi \alpha}\left|X^{N}\left(t^{-}\right)\right| d t \\
& =\beta \frac{e^{2 i \pi \alpha}}{e^{2 i \pi \alpha}+1}(b-a)
\end{aligned}
$$

Hence, one obtains the following result:

$$
K=\pi\left(\frac{1}{2}-\alpha\right) \frac{(b-a)}{\cos (\pi \alpha)}
$$

\section{References}

1. Achenbach JD, Epstein HI (1967) Dynamic interaction of a layer and a half-space. ACSE J Eng Mech Div EM 5:27-42

2. Adams GG (1995) Self-excited oscillations of two elastic halfspaces sliding with a constant coefficient of friction. J Appl Mech 62:867-872

3. Adams GG (1998) Steady sliding of two elastic half-spaces with friction reduction due to interface stick-slip. J Appl Mech 65:470-475

4. Adams GG (2001) An intersonic slip pulse at a frictional interface between dissimilar materials. J Appl Mech 68:81-86

5. Adda-Bedia M, Ben Amar M (2003) Self-sustained slip pulses of finite size between dissimilar materials. J Mech Phys Solids 51:1849-1861

6. Ahn YJ, Bertocchi Barber JR (2008) Shakedown of coupled twodimensional discrete frictional systems. J Mech Phys Solids 56:3433-3440

7. Amontons G (1699) On the resistance originating in machines. Proc Fr R Acad Sci 1732:206-222

8. Andrews DJ, Ben-Zion Y (1997) Wrinkle-like slip pulse on a fault between different materials. J Geophys Res 102:553-571

9. Aubry S (1980) Defectibility and frustration in incommensurate structures: the devil's stair case transformation. Ferroelectrics 24:53-60
10. Ballard P (1999) A counter-example to uniqueness in quasi-static elastic contact problems with small friction. Int $\mathrm{J}$ Eng Sci 37:163-178

11. Ben-Zion Y, Andrews DJ (1998) Properties and implications of dynamic rupture along a material interface. Bull Seismol Soc Amer 88:1085-1094

12. Brochard-Wyart F, de Gennes PG (2007) Naive model for stickslip processes. Eur Phys J E23:439-444

13. Bui HD (2006) Fracture mechanics: inverse problems and solutions. Springer, Berlin

14. Bui HD, Oueslati A (2005) The sliding interface crack with friction between elastic and rigid bodies. J Mech Phys Solids 53:1397-1421

15. Cochard A, Rice JR (2000) Fault rupture between dissimilar materials: ill-posedness, regularization and slip-pulse response. J Geophys Res Solid Earth 105:25891-25907

16. Comninou M, Dundurs J (1977) Elastic interface waves involving separation. J Appl Mech 44:222-226

17. Comninou M, Dundurs J (1978) Elastic interface waves and sliding between two solids. J Appl Mech 45:325-330

18. Caroli C (2000) Slip pulses at a sheared frictional viscoelasticnondeformable interface. Phys Rev E 62:1729-1737

19. Coulomb CA (1785) The theory of simple machines. Mem Math Phys Acad Sci 10:161-331 (in French)

20. Deng X (1994) An asymptotic analysis of stationary and moving cracks with frictional contact along bimaterial and in homogeneous solids. Int J Solids Struct 31:2407-2429

21. Désoyer T, Martins JAC (1998) Surface instabilities in a Mooney-Rivlin body with frictional boundary conditions. Int J Adhes Adhesives 18:413-419

22. Feeny B, Guran A, Hinrichs N, Popp K (1998) A historical review on dry friction and stick-slip phenomena. Appl Mech Rev 51:321-340

23. Freund LB (1978) Discussion: elastic interface waves involving separation. J Appl Mech 45:226-228

24. Klarbring A (1990) Examples of non-uniqueness and non-existence of solutions to quasistatic contact problems with friction. Arch Appl Mech 60:529-541

25. Harris RA, Day SM (1997) Effects of a low-velocity zone on a dynamic rupture. Bull Seismol Soc Am 87:1267-1280

26. Martins JAC, Guimaraes J, Faria LO (1995a) Dynamic surface solutions in linear elasticity and viscoelasticity with frictional boundary conditions. J Vib Acoust 117:445-451

27. Martins JAC, Simões FMF (1995b) On some sources of instability/ill-posedness in elasticity problems with Coulomb's friction. Contact Mech 95-106 (edited by M. Raous)

28. Martins JAC , Barbarin S, Raous M, Pinto da Costa A (1999) Dynamic stability of finite dimensional linearly elastic systems with unilateral contact and Coulomb friction. Comput Methods Appl Mech Eng 177:289-328

29. Martins JAC, Marques MDPM, Gastaldi R (1994) On an example of nonexistence of solution to a quasi-static frictional contact problem. Eur J Mech A Solids 13:113-133

30. Massi F, Baillet L, Giannini O, Sestieri A (2007) Brake squeal: linear and nonlinear numerical approaches. Mech Syst Sig Process 21:2374-2393

31. Moirot F, Nguyen QS (2000b) An example of stick-slip wave. CR Mecanique 328:663-669

32. Moirot F, Nguyen QS (2000a) Brake squeal: A problem of flutter instability of the steady sliding solution? Arch Mech 52:645-662

33. Moirot F, Nguyen QS, Oueslati A (2002) An example of stickslip and stick-slip-separation waves. Eur J Mech A Solids 22:107-118

34. Moirot F (1998) Etude de la stabilité d'un équilibre en présence de frottement de Coulomb: application au crissement des freins à disques. PhD Thesis, Ecole Polytechnique 
35. Mukesh LD, Jonnalagadda KK, Kandikatla RK, Kesava RK (2006) Silo music: sound emission during the flow of granular materials through tubes. Powder Technol 167:55-71

36. Murty GS (1975) Wave propagation at an unbonded interface between two elastic half-spaces. J Acoustic Soc Am 58:1094-1095

37. Muskhelishvili NI (1953) Some basic problems of the mathematical theory of elasticity. Noordhoff, Groningen

38. Nakai M, Yokoi M (1996) Band brake squeal. J Vib Acoustics 118:190-197

39. Nguyen QS, Oueslati A, Steindl A, Teufel A, Troger H (2008) Travelling interface waves in a brake-like system under unilateral contact and Coulomb friction. CR Mecanique 336:203-209

40. Oancea V, Laursen TA (1997) Stability analysis of state-dependent dynamic frictional sliding. Int J Nonlinear Mech 32:837-853

41. Oestreich M, Hinrichs N, Popp K (1996) Bifurcation and stability analysis for a nonsmooth friction oscillator. Arch Appl Mech 66:301-314

42. Oueslati A, Nguyen QS, Baillet L (2003) Stick-slip-separation waves in unilateral and frictional contact. $\mathrm{CR}$ Mecanique 331:133-140

43. Oueslati A (2004) Ondes élastiques de surface et fissures d'interface sous contact unilatéral et frottement de Coulomb. PhD Thesis, Ecole Polytechnique, Paris
44. Prakash V (1998) Frictional response of sliding interfaces subjected to time varying normal pressures. J Tribol 120:97-102

45. Radok JMR (1956) On the solution of problems of dynamic plane elasticity. Q Appl Math 14:289

46. Ranjith K, Rice JR (2001) Slip dynamics at an interface between dissimilar materials. J Mech Phys Solids 49:341-361

47. Renardy M (1992) Ill-posedness at the boundary for elastic solids sliding under Coulomb friction. J Elasticity 27:281-287

48. Simoes FM, Martins JAC (1998) Instability and ill-posedness in some friction problems. Int J Eng Sci 36:1265-1293

49. Schallamach A (1971) How does rubber slide? Wear 17:301-312

50. Vola D, Raous M, Martins JAC (1999) Friction and instability of steady sliding: squeal of a rubber/glass contact squeal. Int J Num Meth Eng 46:1699-1720

51. Weertman J (1963) Dislocations moving uniformly on the interface between isotropic media of different elastic properties. J Mech Phys Solids 11:197-204

52. Zharii OY (1996) Frictional contact between the surface wave and a rigid strip. J Appl Mech 63:15-20 